\title{
Effect of prosthetic gel liner thickness on gait biomechanics and pressure distribution within the transtibial socket
}

\author{
Erin Boutwell, MS; ${ }^{1-2 *}$ Rebecca Stine, MS; ${ }^{1,3}$ Andrew Hansen, PhD; ${ }^{1,4}$ Kerice Tucker, BS; ${ }^{1}$ Steven Gard, PhD ${ }^{1-3}$ \\ ${ }^{1}$ Northwestern University Prosthetics-Orthotics Center for Education and Research, Department of Physical Medicine \\ and Rehabilitation, Feinberg School of Medicine, Northwestern University, Chicago, IL; ${ }^{2}$ Department of Biomedical \\ Engineering, McCormick School of Engineering and Applied Science, Northwestern University, Evanston, IL; ${ }^{3}$ Jesse \\ Brown Department of Veterans Affairs (VA) Medical Center, Chicago, IL; ${ }^{4}$ Minneapolis VA Health Care System, \\ Minneapolis, $M N$
}

\begin{abstract}
Prosthetic gel liners are often prescribed for persons with lower-limb amputations to make the prosthetic socket more comfortable. However, their effects on residual limb pressures and gait characteristics have not been thoroughly explored. This study investigated the effects of gel liner thickness on peak socket pressures and gait patterns of persons with unilateral transtibial amputations. Pressure and quantitative gait data were acquired while subjects walked on liners of two different uniform thicknesses. Fibular head peak pressures were reduced $(p=0.04)$ with the thicker liner by an average of $26+/-$ $21 \%$, while the vertical ground reaction force (GRF) loading peak increased $3+/-3 \%(p=0.02)$. Most subjects perceived increased comfort within the prosthetic socket with the thicker liner, which may be associated with the reduced fibular head peak pressures. Additionally, while the thicker liner presumably increased comfort by providing a more compliant limb-socket interface, the higher compliance may have reduced force and vibration feedback to the residual limb and contributed to the larger vertical GRF loading peaks. We conclude that determining optimal gel liner thickness for a particular individual will require further investigations to better identify and understand the compromises that occur between user perception, residuallimb pressure distribution, and gait biomechanics.
\end{abstract}

Key words: artificial limbs, gait analysis, gel liner, interface pressure, pin suspension, pressure sensors, prosthesis, rehabilitation, residual limb, transtibial amputee.

\section{INTRODUCTION}

Possibly the single most critical aspect of any prosthesis is the quality of the interface between the residual limb and the prosthesis [1]. Prosthetic gel liners are often prescribed for persons with lower-limb amputations to provide comfort and cushioning to the residual limb within the prosthetic socket. The increased compliance provided by the gel liners may reduce shear stresses between the prosthetic socket and the skin, create a more

\footnotetext{
Abbreviations: $\mathrm{ARL}=$ average residual limb, $\mathrm{BRL}=$ bony residual limb, ESAR = energy-storage-and-return, GRF = ground reaction force, JBVAMC = Jesse Brown VA Medical Center, MARL = Motion Analysis Research Laboratory, PRL = padded residual limb, PTB = patellar-tendon-bearing, $\mathrm{SACH}=$ solidankle cushioned heel, $\mathrm{SD}=$ standard deviation, $\mathrm{SDE}=$ distal end of the tibia sensor, $\mathrm{sDT}=$ distal anterior tibia sensor, $\mathrm{sFH}=$ fibular head sensor, $\mathrm{sMG}=$ medial gastrocnemius sensor, $\mathrm{sPT}=$ patellar tendon sensor, TSB = total-surface-bearing, VA = Department of Veterans Affairs.

*Address all correspondence to Erin Boutwell, MS; Northwestern University Prosthetics-Orthotics Center, 680 N Lake Shore Dr, Suite 1100, Chicago, IL 60611; 312-5035700; fax: 312-503-5760.
}

Email: erinboutwell2015@u.northwestern.edu http://dx.doi.org/10.1682/JRRD.2010.06.0121 
uniform pressure distribution over the residual limb, and contribute to shock absorption during walking.

Shock absorption is required during the loading response phase of gait as load is transferred from the trailing to the leading leg. The provision of adequate shock absorption is a critical concern in lower-limb prostheses. With every step, forces are transmitted through the prosthesis to the residual limb and proximal joints. Insufficient shock attenuation has previously been associated with low back pain [2] and joint degeneration/ osteoarthritis [3]. Also, high-impact forces associated with repeated foot-ground contact may contribute to the high incidence of residual-limb pain [4].

Shock absorption during both nondisabled and amputee gait is provided by compliant mechanisms within the locomotor system. The anatomical heel pad and plantarflexion of the ankle, two shock-absorbing mechanisms in nondisabled persons, are not present after transtibial amputation. Additionally, stance-phase knee flexion and pelvic obliquity also provide substantial shock absorption during nondisabled walking [5-6]. Although individuals with unilateral transtibial amputations are able to utilize these mechanisms, their magnitudes are typically reduced and the amputees' patterns of movement are often altered compared with nondisabled individuals [7-8], suggesting a reduction in the capacity to absorb shock. However, in lower-limb amputees, the compliance of residual limb soft tissue that interfaces with the prosthetic socket probably provides some degree of shock absorption in the residual limb-prosthesis system, but this compliance may not be sufficient to compensate for the loss or reduction of the anatomical shockabsorbing mechanisms. The subsequent decrease in shock absorption may be partially responsible for the pain and discomfort at the residual limb-prosthetic socket interface that is reported by many prosthesis users.

Components such as shock-absorbing pylons and compliant foot-ankle devices have been designed to artificially introduce compliance into the prosthesis itself, potentially contributing to a reduction in shock forces during walking. Also, many energy-storage-and-return (ESAR) feet increase prosthetic compliance, compressing upon initial contact with the ground and potentially absorbing or dissipating energy associated with this impact. The effects of different prosthetic feet and shockabsorbing pylons have been evaluated using walking speed, step length, stance-phase duration, and ground reaction force (GRF) profiles [9-14]. However, no con- sistent effects on walking speed or GRF peaks have been reported with either type of component. Casillas et al. observed an increase in walking speed with an ESAR foot compared with a solid-ankle cushioned heel (SACH) foot [9], but Lehmann et al. [10] and Torburn et al. [11] both reported no changes in walking speed between ESAR and SACH feet. Additionally, although Barr et al. reported that the vertical GRF peak during prostheticside weight acceptance had a reduced magnitude and occurred sooner after initial contact with an ESAR foot than with the SACH foot [12], Lehmann et al. observed no significant differences in a similar comparison [10]. Studies that have compared shock-absorbing pylons to rigid pylons generally have reported few differences in either walking speeds or vertical GRFs when subjects walked at a self-selected speed [13-14].

Gel liners may serve as yet another means of restoring shock absorption during gait. They are recognized as an important factor in altering pressure distribution within the socket [15]. The gel used within prosthetic liners-often silicone or other elastomeric materialsincreases compliance at the residual limb-prosthetic socket interface, reducing local peak pressures and creating a more uniform distribution of pressure over the residual limb. However, discomfort within the prosthetic socket may also be caused by sweating and rashes, both of which have been associated with gel liner use [16]. The greater thickness of the gel liner may increase the temperature at the limb-socket interface, leading to increased incidences of perspiration and associated skin irritation. Thus, a balance should be sought between increased comfort caused by the presumed lower peak pressure transmission and potentially increased incidences of skin irritation.

Several studies have investigated pressures at the limb-socket interface [17-22]. These studies have evaluated interface pressures with patellar-tendon-bearing (PTB) sockets [17-18,20-21], an unrectified hydrocast socket [17], and a total-surface-bearing (TSB) socket [19]. Furthermore, these studies determined how interface stresses may be affected by a variety of factors, including cadence, prosthetic feet, prosthetic pylons, time between sessions, time of day, socket type, and activity type [17-19,21-22]. Of these factors, time between measurements and socket type appear to be the most influential in altering interface pressure distribution [19,21-22]. Research regarding the effect of gel liners on peak pressures at the limb-socket interface has been limited. Sonck 
et al. used solid-state force transducers to measure pressure between the residual limb and the interface material in 23 subjects with unilateral transtibial amputation [20]. Socket type was not specified, nor was the thickness of the gel liner indicated. The investigators reported that a silicone gel liner reduced average peak pressures over the patellar tendon and medial and lateral tibial condyles when compared with Kem-Blo, a conventional spongerubber material. Because the peak pressures recorded with the gel liner were consistently lower, Sonck et al. concluded that the function of the gel may be to achieve a more uniform pressure distribution over the surface of the residual limb. The effect of the thickness of the gel liner on peak pressures within the socket has not been previously evaluated. For clinical consideration, the ordering instructions for Ohio Willow Wood Alpha ${ }^{\circledR}$ liners (Mt. Sterling, Ohio) suggest that prosthetists should fit the thickest liner that is comfortable for the patient. Currently, ways in which peak pressure distributions may be altered by the introduction of gel liners of different thickness is unknown.

This study evaluated the effect of liner thickness on pressure distributions at select sites on the residual limb and on gait characteristics of persons with unilateral transtibial amputations. Within this article, the term "pressure" is used to designate normal stress, or stress perpendicular to the surface of the residual limb [23]. We hypothesized that a thicker gel liner would reduce local peak pressures and create a more uniform distribution of pressures at the residual limb-liner interface. This would represent a more homogeneous distribution of pressure across the residual limb, which is the objective of the TSB socket used within this study. We also thought that the additional compliance provided by the thicker gel liner would increase the shock absorption of the prosthetic system, thereby reducing the magnitude of the peak forces transmitted to the residual limb during gait. Thus, we hypothesized that prosthetic-side vertical and fore-aft GRF weight-acceptance peaks would be reduced and occur later after initial contact in the thicker liner condition. As a consequence of this improved shock absorption, we anticipated that subjects might respond by increasing their freely selected walking speed without experiencing a resultant increase in transmitted impact force. To test these hypotheses, we recorded and analyzed real-time measurements of pressure on the residual limb, as well as kinematic and kinetic data, for subjects walking on level ground wearing each of two gel liners: a thin $3 \mathrm{~mm}$ liner and a thick $9 \mathrm{~mm}$ liner.

\section{METHODS}

Subjects with a unilateral transtibial amputation were recruited for participation in this study. Inclusion criteria were as follows: all subjects were between the ages of 18 and 70, had an amputation without serious complications, and had at least 6 months experience using a definitive prosthesis. Subjects with concurrent medical issues or who were prescribed medication that could significantly interfere with balance or gait were excluded from the study. Additionally, subjects had to be able to safely walk at least $10 \mathrm{~m}$ over level ground without the use of an assistive device.

Two Alpha ${ }^{\circledR}$ prosthetic gel liners-a thin $3 \mathrm{~mm}$ liner and a thick $9 \mathrm{~mm}$ liner-were tested as subjects walked at a self-selected walking speed. Both liners were of uniform thickness and had a small umbrella on the distal end that provided a somewhat more rigid interface for the shuttlelock pin. The "uniform" version of the $9 \mathrm{~mm}$ liner included thinning of the posterior wall behind the knee to permit knee flexion. Pressure sensors were placed over five anatomical locations on the residual limb. These sensors were labeled as follows: patellar tendon sensor (sPT), distal anterior tibia sensor (sDT), distal end of the tibia sensor (sDE), fibular head sensor ( $\mathrm{sFH}$ ), and medial gastrocnemius sensor (sMG). The locations of the pressure sensors on the residual limb are shown in Figure 1. The locations of the sFH, sPT, and sDE were determined through palpation of the fibular head, patellar tendon, and distal end of the tibia, respectively, and the center of the sensor array was placed directly over the landmark. The sMG was large enough to encompass almost the entire posterior aspect of the residual limb. The distal anterior tibia pressure was recorded by placing a sensor array across the tibia immediately distal to the SPT. The sensor arrays were positioned by the same investigator for all data collections to provide consistency between data collection sessions and subjects. The sensor array sizes were $6.0 \times 3.0 \mathrm{~cm}(\mathrm{sPT}, \mathrm{sDT}, \mathrm{sFH}), 2.0 \times 2.0 \mathrm{~cm}$ (sDE), and $15.0 \times 6.0 \mathrm{~cm}(\mathrm{sMG})$; the sensors had an individual surface area of $1.0 \mathrm{~cm}^{2}$, had a thickness of $1.0 \mathrm{~mm}$, and were coated in thermoplastic polyurethane film. The tape used to secure the sensors onto the residual limb was 3M Micropore paper tape (3M; St. Paul, Minnesota) with a thickness of $0.12 \mathrm{~mm}$. All data collection occurred in the Jesse Brown Department of Veterans Affairs (VA) Medical Center (JBVAMC) Motion Analysis Research Laboratory 


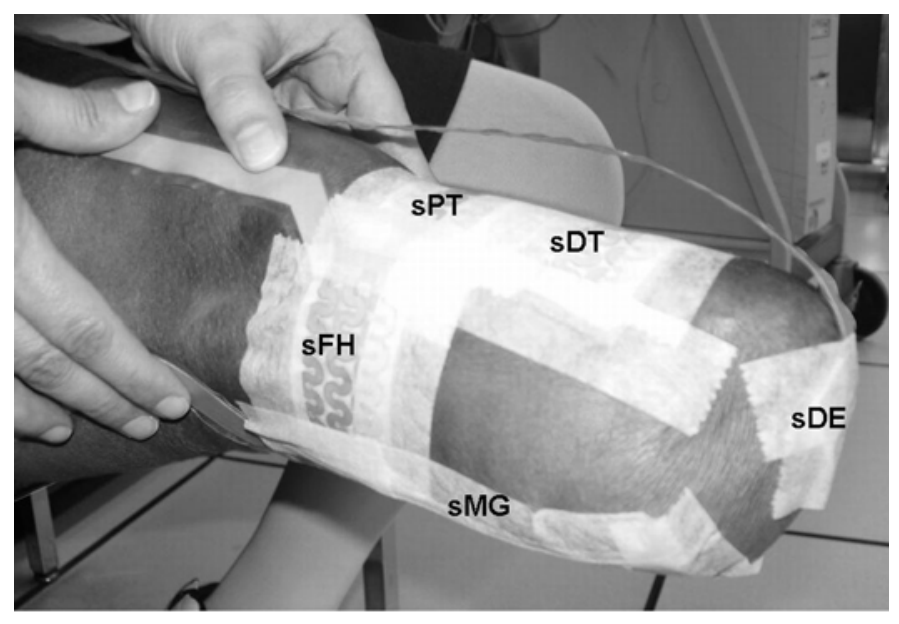

Figure 1.

Pressure sensor placement on residual limb. Sensors are labeled SPT (patellar tendon), SDT (distal anterior tibia), SDE (distal end of the tibia), SFH (fibular head), and SMG (medial gastrocnemius). Only data from sPT, sDT, and sFH were analyzed for all subjects.

(MARL), which is equipped with an eight-camera Eagle Digital RealTime system (Motion Analysis Corporation; Santa Rosa, California), six force platforms (AMTI; Watertown, Massachusetts), and a Novel pliance ${ }^{\circledR}$ pressure sensor system (Novel Electronics; Munich, Germany).

Two experimental prostheses were fabricated for each subject. Each experimental prosthesis was comprised of an Otto Bock 1D35 prosthetic foot (Duderstadt, Germany), a rigid aluminum pylon, the liner, and a TSB socket fabricated for the appropriate liner. The socket, pylon, and foot were connected using standard pyramid adapters. Sockets were fabricated by digitizing the subject's residual limb as the liner was worn using an Omega Tracer ${ }^{\circledR}$ system (Ohio Willow Wood) while the limb was held horizontally. A certified prosthetist then performed a global reduction of 5-10 mm (3 mm liner) or 15-20 mm (9 $\mathrm{mm}$ liner) based on recommendations from the Tracer's manufacturer. The modified socket was fabricated using Squirt Shape technology previously developed in our laboratory [24] to create a polypropylene socket with a $4 \mathrm{~mm}$ uniform thickness. Trimlines were created at the direction of the prosthetist, and then subjects were fitted with the socket. The prosthetist modified the socket as necessary if the socket fit was not appropriate. If a suitable socket fit could still not be achieved after modification by the prosthetist, the digitization process was repeated and a new socket was fabricated. Initially, all subjects were fitted with a prosthetic socket to accommodate the $3 \mathrm{~mm}$ liner. However, issues with socket fitting required some subjects to begin the study with the $9 \mathrm{~mm}$ liner. Once their sockets were comfortably fitted, subjects were given an accommodation period of at least 2 weeks on each experimental prosthesis prior to data collection. Subjects were instructed to wear the same shoes to both data collection sessions for consistency, and all data collections were performed at the same time of day for each subject. To minimize the effects of longterm changes on limb-socket interface pressure, the investigators attempted to ensure that the second data collection occurred no more than 8 weeks after the first data collection session.

Reflective markers were placed on the subject in a modified Helen Hayes arrangement [25]. Markers on the prosthesis were placed on the lateral and medial surfaces of the socket at the anatomical knee axis of rotation, the superior aspect of the shoe at the estimated metatarsophalangeal joint of the dorsum of the prosthetic foot, the heel of the shoe at the same elevation from the ground as the dorsal foot marker, and the lateral and medial surfaces of the prosthetic foot at the approximate location of the intact ankle axis of rotation. Pressure sensor arrays were taped to the residual limb, and then the gel liner was rolled over the sensors. Once instrumented, subjects were instructed to walk down the center of a $10 \mathrm{~m}$ walkway at their freely selected speed. Trials were collected until five clean force plate strikes (i.e., a foot placement within the borders of a single plate with no contralateral foot contact) were obtained for each foot. After testing both liners, subjects completed a questionnaire to compare the $9 \mathrm{~mm}$ liner condition to the $3 \mathrm{~mm}$ liner condition. This questionnaire consisted of 19 statements relating to liner comfort and function that subjects rated using a 5-point Likert scale. Additionally, subjects were asked to indicate which liner they preferred. This questionnaire was not validated but was administered to provide some indication of differences in the subjects' perceptions between the two liner conditions.

Marker data from the kinematic data collection were bidirectionally filtered with a fourth-order Butterworth filter having an effective cutoff frequency of $6.0 \mathrm{~Hz}$ using EVa RealTime software (Motion Analysis Corporation). The data files were then processed using OrthoTrak software (Motion Analysis Corporation) to calculate kinematic, kinetic, and temporal-spatial data based on a link-segment 
model of the body. The joint angles and GRFs, as well as the peak pressure values from each sensor array captured with the pliance system, were further analyzed in MATLAB (MathWorks Inc; Natick, Massachusetts). Data were plotted against percentage of gait cycle and averaged between trials for each subject. To ensure identification of those peak pressures associated with shock absorption during loading response phase, we analyzed the magnitudes of peak pressures at each of the measurement sites during the first 40 percent of stance phase. Similarly, the vertical and fore-aft GRFs during loading response phase were analyzed to determine the magnitude of the peaks and the time of occurrence relative to initial contact of the foot. Stancephase knee flexion and pelvic obliquity range of motion were determined from the kinematic data. The mean and standard deviation (SD) of variables of interest were calculated for the individual subjects, the overall group of subjects, as well as for two subgroups categorized according to residual limb type.

Data were analyzed for statistical significance using SPSS (SPSS Inc; Chicago, Illinois). For data sets that fulfilled normality assumptions, a paired $t$-test was performed to determine statistically significant changes attributable to liner thickness. Data sets that violated the assumption of normality were analyzed by nonparametric means (i.e., Wilcoxon signed-ranks test). All $p$-values presented in this article are from the paired $t$-test analysis unless otherwise noted. Changes in variables were considered statistically significant at the $p<0.05$ level.

\section{RESULTS}

Eleven of twelve subjects completed this study; one subject was lost to follow-up between the two data collection sessions. Subject information, including amputation etiology, time since amputation, and familiarity with liner use, is presented in Table 1. During data collection, it became apparent that the two liners affected subjects with varied residual limb types differently. Therefore, the study prosthetist subjectively categorized subjects as either 'bony,' 'average,' or 'padded' depending on the amount of soft tissue on the residual limb. This classification involved the assessment of limb characteristics such as limb shape and length, muscle strength, knee joint structure, and surgical technique. The bony residual limb (BRL) group consisted of five subjects ( 2 males, 3 females) with a mean \pm SD age of $58.2 \pm 9.9 \mathrm{yr}$, height of $169.2 \pm 11.6 \mathrm{~cm}$, and mass of $77.5 \pm 9.7 \mathrm{~kg}$. The padded residual limb (PRL) group consisted of five subjects (2 males, 3 females) with a mean \pm SD age of $52.4 \pm 8.3 \mathrm{yr}$, height of $174.5 \pm 9.9 \mathrm{~cm}$, and mass of $101.2 \pm 18.5 \mathrm{~kg}$. The average residual limb (ARL) category consisted of a single 62 yr old female subject (height $177 \mathrm{~cm}$; mass $76.4 \mathrm{~kg}$ ). Seven subjects wore the $3 \mathrm{~mm}$ liner and socket for the first data collection

Table 1.

Subject information.

\begin{tabular}{|c|c|c|c|c|c|c|c|c|c|}
\hline Subject & Sex & $\begin{array}{l}\text { Age } \\
\text { (yr) }\end{array}$ & $\begin{array}{c}\text { Height } \\
\text { (cm) }\end{array}$ & $\begin{array}{c}\text { Mass } \\
(\mathbf{k g})\end{array}$ & $\begin{array}{c}\text { Gel Liner in } \\
\text { Current Prosthesis? } \\
(\mathbf{m m})\end{array}$ & $\begin{array}{c}\text { Amputation } \\
\text { Etiology }\end{array}$ & $\begin{array}{c}\text { Time Since } \\
\text { Amputation } \\
\text { (yr) }\end{array}$ & $\begin{array}{l}\text { Limb } \\
\text { Type }\end{array}$ & $\begin{array}{l}\text { First Tested } \\
\text { Liner } \\
(\mathrm{mm})\end{array}$ \\
\hline 1 & $\mathrm{M}$ & 64 & 187.5 & 90.5 & No & Trauma & 32 & Bony & 9 \\
\hline 2 & M & 43 & 182.0 & 96.0 & 6 & Trauma & 7 & Padded & 3 \\
\hline 3 & M & 49 & 183.0 & 105.5 & 6 & Disease $^{*}$ & 2 & Padded & 9 \\
\hline 4 & M & 67 & 167.5 & 74.5 & 9 & Trauma & 13 & Bony & 3 \\
\hline 7 & $\mathrm{~F}$ & 46 & 172.0 & 65.0 & 6 & Trauma & 10 & Bony & 3 \\
\hline 8 & $\mathrm{~F}$ & 59 & 162.0 & 88.5 & No & Trauma & 33 & Padded & 3 \\
\hline 9 & $\mathrm{~F}$ & 63 & 180.0 & 131.1 & Yes & PVD & 5 & Padded & 3 \\
\hline 10 & $\mathrm{~F}$ & 49 & 158.0 & 83.0 & 6 & Disease $^{*}$ & 7 & Bony & 9 \\
\hline
\end{tabular}


session, while four subjects (2 BRL, 2 PRL) completed their first data collections on the $9 \mathrm{~mm}$ liner and socket.

Two of the pressure sensors - the sDE and sMGwere damaged after the first few data collection sessions and were removed from the protocol. Therefore, peak pressure data are only available for the sPT, sDT, and sFH. Representative plots of peak pressure values during stance phase are provided in Figure 2. Furthermore, for some subjects, one or more of the remaining sensors saturated during walking trials, so these data were excluded from the pressure analysis. Two subjects, including the ARL subject and one PRL subject, experienced saturation at all sensor locations; one BRL subject had a saturated SPT; and one PRL subject experienced saturation of the $\mathrm{sFH}$ and sDT.

Peak pressure values for each subject are provided in Table 2. These pressures correspond to the maximum pressure achieved during weight acceptance on the prosthetic limb and were recorded over a minimum of seven steps. The one exception was Subject 6's $9 \mathrm{~mm}$ sPT data, in which only three steps of data were available. A significant reduction in peak pressure over the $\mathrm{sFH}$ was observed for all subjects $(p=0.04)$. However, no statistically significant changes in peak pressures were detected between liner conditions for either the sPT or sDT locations.

No differences between liners were found in the magnitudes of prosthetic-side stance-phase knee flexion or pelvic obliquity, nor were any differences observed in the peak fore-aft GRF during weight acceptance (Table 3). The timing of the peak vertical GRF during weight acceptance was also unchanged, but there was a statistically significant increase in the magnitude of this peak for the $9 \mathrm{~mm}$ liner condition ( $p=0.02$, Wilcoxon signed-ranks test). Peak vertical GRF magnitudes are provided in Table 4. Figure 3 illustrates representative vertical GRF curves for one subject using the two different liners. Finally, the mean freely selected walking speeds were $1.11 \pm 0.17 \mathrm{~m} / \mathrm{s}$ with the $3 \mathrm{~mm}$ liner and $1.14 \pm 0.19 \mathrm{~m} / \mathrm{s}$ with the $9 \mathrm{~mm}$ liner. The difference between walking speeds with the two liners was not statistically significant.

The questionnaire results (Figure 4) indicated differences in subject perception between the two liner conditions. Out of the five BRL subjects, four preferred the $9 \mathrm{~mm}$ liner, indicating that it increased comfort during standing and walking, reduced pain in individual areas on the residual limb, and generally reduced pain at residual limb-socket interface. Conversely, only two of the five
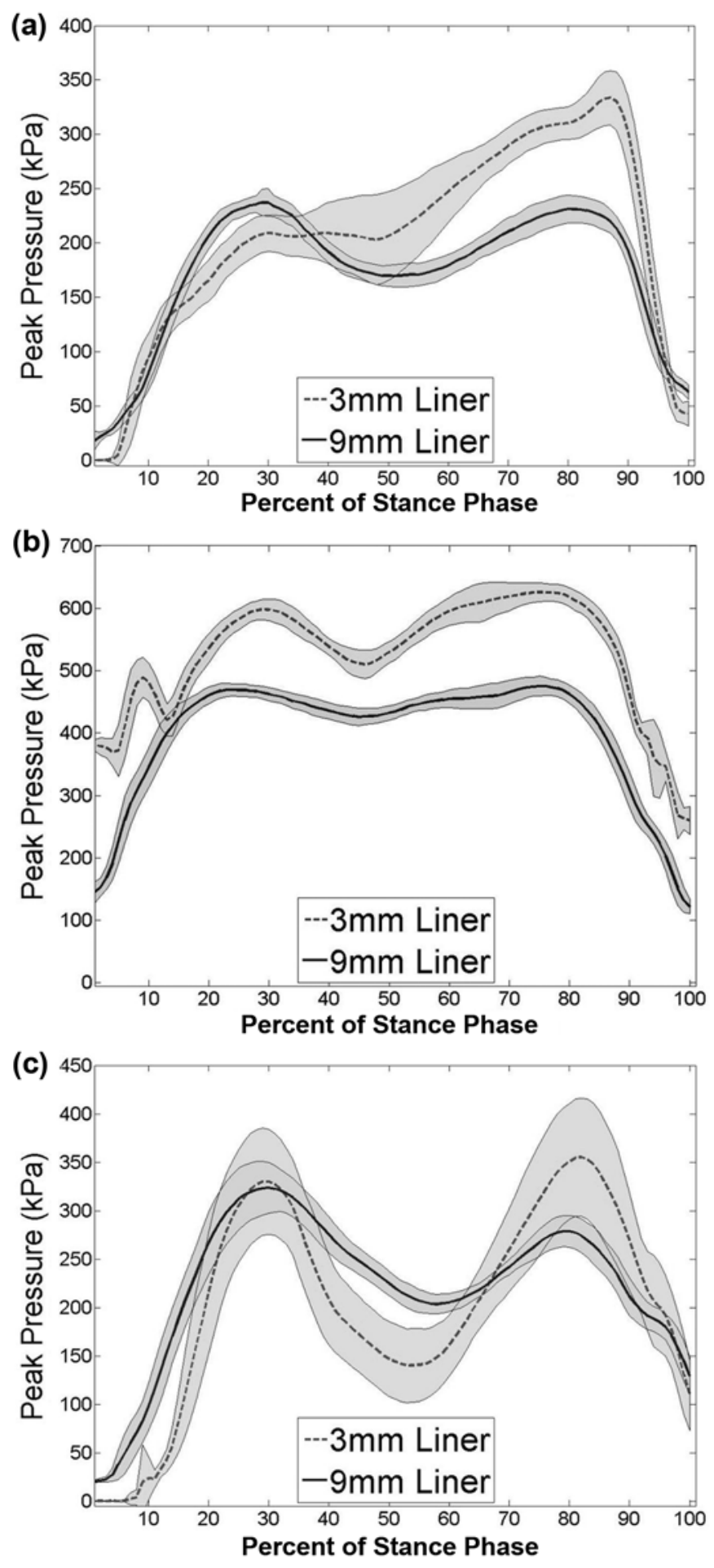

Figure 2.

Representative peak pressure curves for single subject for (a) patellar tendon, (b) fibular head, and (c) distal anterior tibia pressure sensor locations. These curves represent peak pressures averaged over 13 (3 mm liner) and 15 (9 mm liner) steps. Shading represents \pm 1 standard deviation. 
Table 2.

Mean stance-phase peak pressures (kilopascals) during loading ( $0 \%-40 \%$ stance phase) at freely selected walking speeds for each subject and limb-type group. Bold typeface indicates statistical significance at $p<0.05$. Data presented as mean \pm standard deviation.

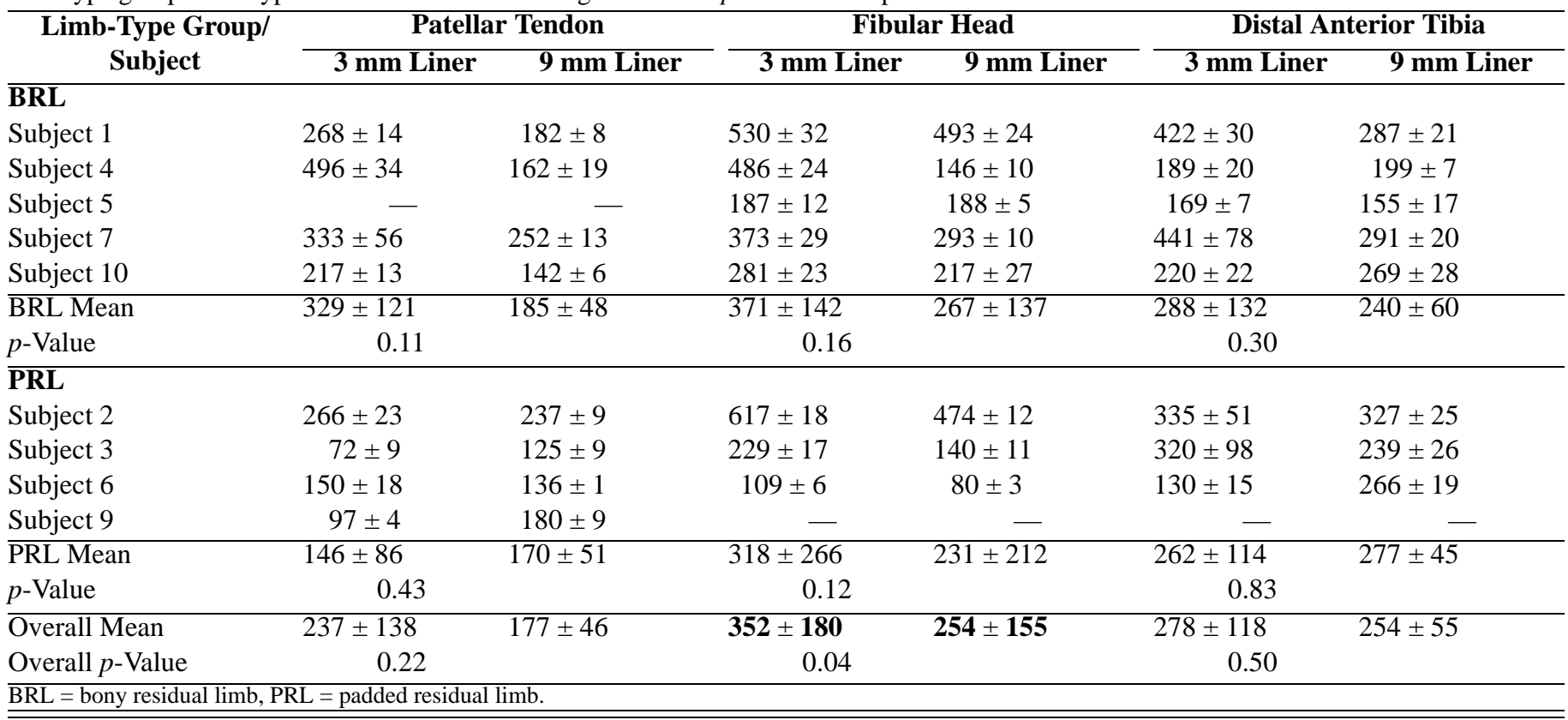

Table 3.

Average walking speed and prosthetic-side gait parameters at freely selected walking speeds. Bold typeface indicates statistical significance at $p<$ 0.05. Data presented as mean \pm standard deviation.

\begin{tabular}{|c|c|c|c|}
\hline Parameter & 3 mm Liner & 9 mm Liner & p-Value \\
\hline \multicolumn{4}{|l|}{ Bony Residual Limb } \\
\hline Walking Speed (m/s) & $1.04 \pm 0.13$ & $1.03 \pm 0.11$ & 0.50 \\
\hline Vertical GRF Loading Peak (\% BW) & $98.9 \pm 3.3$ & $103.4 \pm 5.2$ & 0.06 \\
\hline Fore-aft GRF Braking Peak (\% BW) & $10.4 \pm 2.9$ & $10.1 \pm 3.7$ & 0.78 \\
\hline Timing of Fore-aft GRF Braking Peak (\% GC) & $20.2 \pm 4.9$ & $28.0 \pm 7.5$ & 0.11 \\
\hline \multicolumn{4}{|l|}{ Padded Residual Limb } \\
\hline Walking Speed (m/s) & $1.11 \pm 0.15$ & $1.17 \pm 0.14$ & 0.05 \\
\hline Vertical GRF Loading Peak (\% BW) & $108.4 \pm 13.0$ & $110.7 \pm 13.0$ & 0.11 \\
\hline Timing of Vertical GRF Loading Peak (\% GC) & $32.8 \pm 8.3$ & $32.0 \pm 7.8$ & 0.37 \\
\hline Fore-aft GRF Braking Peak (\% BW) & $10.2 \pm 2.6$ & $10.6 \pm 2.0$ & 0.62 \\
\hline Timing of Fore-aft GRF Braking Peak (\% GC) & $23.6 \pm 3.4$ & $24.0 \pm 4.1$ & 0.87 \\
\hline Walking Speed (m/s) & $1.11 \pm 0.17$ & $1.14 \pm 0.19$ & 0.10 \\
\hline Vertical GRF Loading Peak (\% BW) & $103.9 \pm 9.7$ & $106.9 \pm 9.6$ & $0.02^{*}$ \\
\hline Timing of Vertical GRF Loading Peak (\% GC) & $34.4 \pm 7.0$ & $32.6 \pm 5.3$ & 0.26 \\
\hline Fore-aft GRF Braking Peak (\% BW) & $10.5 \pm 2.5$ & $10.8 \pm 3.1$ & 0.63 \\
\hline Timing of Fore-aft GRF Braking Peak (\% GC) & $21.5 \pm 4.3$ & $25.4 \pm 6.1$ & 0.11 \\
\hline Stance-phase Knee Flexion $\left(^{\circ}\right)$ & $10.6 \pm 7.1$ & $12.4 \pm 6.1$ & $0.39^{*}$ \\
\hline Pelvic Obliquity ROM $\left(^{\circ}\right)$ & $5.3 \pm 1.8$ & $5.4 \pm 1.9$ & 0.83 \\
\hline
\end{tabular}

${ }^{*} p$-Values determined by Wilcoxon signed-ranks test.

$\mathrm{BW}$ = body weight, GC = gait cycle, GRF = ground reaction force, $\mathrm{ROM}=$ range of motion . 
Table 4.

Vertical ground reaction force loading peak magnitudes for each subject and limb-type group. Bold typeface indicates statistical significance at $p<0.05$. Data presented as mean \pm standard deviation.

\begin{tabular}{|c|c|c|c|}
\hline $\begin{array}{l}\text { Vertical Ground } \\
\text { Reaction Force } \\
\text { Loading Peaks } \\
\qquad(\times \mathrm{BW})\end{array}$ & $\begin{array}{l}3 \text { mm } \\
\text { Liner }\end{array}$ & $\begin{array}{l}9 \text { mm } \\
\text { Liner }\end{array}$ & $p$-Value \\
\hline \multicolumn{4}{|l|}{$\overline{\text { BRL }}$} \\
\hline Subject 1 & $0.99 \pm 0.02$ & $1.0 \oplus .03$ & 0.06 \\
\hline Subject 4 & $0.96 \pm 0.02$ & $1.0 \notin .06$ & \\
\hline Subject 5 & $1.03 \pm 0.02$ & $1.1 \oplus .01$ & \\
\hline Subject 7 & $1.01 \pm 0.02$ & $1.0 € .03$ & \\
\hline Subject 10 & $0.96 \pm 0.03$ & $0.9 \oplus .04$ & \\
\hline BRL Mean & $0.99 \pm 0.03$ & $1.0 \notin .05$ & \\
\hline \multicolumn{4}{|l|}{ PRL } \\
\hline Subject 2 & $1.21 \pm 0.03$ & $1.2 \oplus .05$ & 0.11 \\
\hline Subject 3 & $1.23 \pm 0.05$ & $1.2 \notin .02$ & \\
\hline Subject 6 & $0.98 \pm 0.02$ & $1.0 \% .02$ & \\
\hline Subject 8 & $1.06 \pm 0.06$ & $1.1 \oplus .03$ & \\
\hline Subject 9 & $0.95 \pm 0.02$ & $0.9 \notin .02$ & \\
\hline PRL Mean & $1.08 \pm 0.13$ & $1.1 \oplus .13$ & \\
\hline \multicolumn{4}{|l|}{ Average Residual Limb } \\
\hline Subject 11 & $1.06 \pm 0.03$ & $1.0 \oplus .02$ & - \\
\hline Overall Mean & $1.04 \pm 0.10$ & $1.0 \# .10$ & $0.02^{*}$ \\
\hline
\end{tabular}

PRL subjects preferred the $9 \mathrm{~mm}$ liner. The majority of PRL subjects indicated that they perceived less pistoning and lower energy expenditure while walking with the $9 \mathrm{~mm}$ liner, but they also perceived that the prosthesis was heavier and that their knee was more difficult to flex.

\section{DISCUSSION}

We hypothesized that a thicker gel liner would create a more uniform distribution of pressure over the residual limbs of unilateral, transtibial amputees wearing TSB sockets during walking compared with the pressure distribution created with a thinner gel liner. Specifically, the thicker gel liner was expected to reduce peak pressures over those areas of the anatomy that are regarded as sustaining higher pressures during load-bearing through the prosthesis. As a result of the increased compliance between the prosthesis user and the prosthesis, we anticipated that shock absorption would be improved, resulting

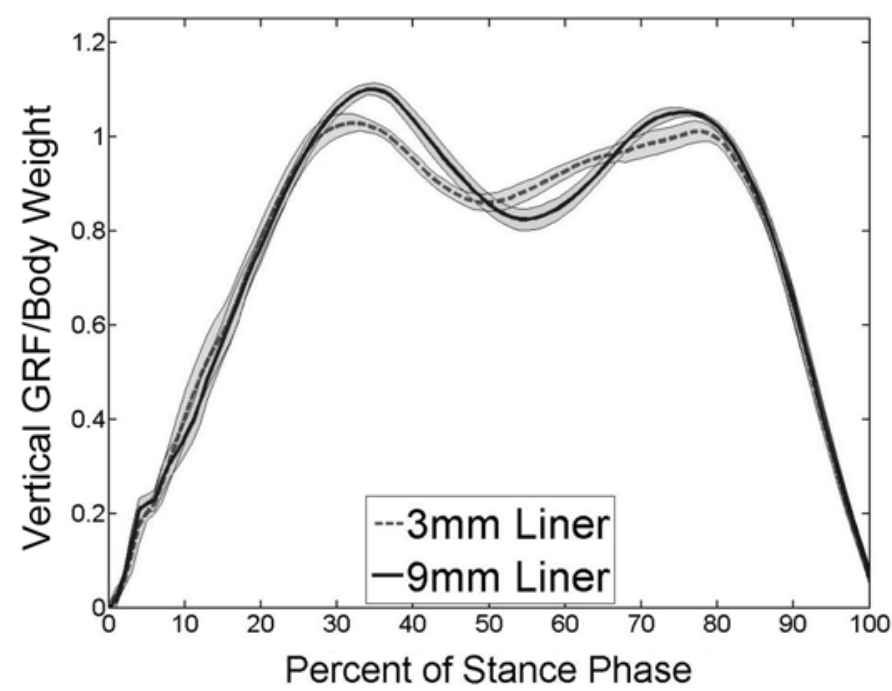

Figure 3.

Representative prosthetic-side vertical ground reaction force (GRF) curves normalized by body weight for single subject at self-selected walking speed. Shading represents \pm 1 standard deviation.

in lower peak GRF magnitudes during weight acceptance. Analysis of the group data indicated that a statistically significant reduction in peak pressure occurred with the $9 \mathrm{~mm}$ gel liner at the sFH only (Table 2), one of the three measurement sites for which pressure data were successfully obtained. In a previous study that measured sock-socket pressures within PTB sockets as subjects walked with various combinations of prosthetic feet and pylons, the investigators reported that changing prosthetic components within a single testing session altered the interface stress by an average of 14.5 percent [21]. Therefore, the 26 percent decrease in mean peak pressure over the $\mathrm{sFH}$ that was observed in this current study supports the assertion that the thickness of a prosthetic gel liner can reduce pressures over bony prominences on the residual limb during loading, as was suggested by previous investigations $[15,20]$.

The mean peak pressures $(180-350 \mathrm{kPa})$ recorded within this study are somewhat higher than those reported in the literature, which range from $55-200 \mathrm{kPa}$ [18-21]. However, three of these four previous investigations were conducted with PTB sockets rather than with the TSB socket design used in this current study. Additionally, two of these studies presented peak pressures averaged over stance phase and thus did not limit their analysis to the loading response phase of gait $[18,20]$. In the single previous study in which a TSB socket design 


\begin{tabular}{|c|c|c|c|c|c|c|c|c|c|c|}
\hline \multirow{2}{*}{ Questionnaire Statement } & \multicolumn{5}{|c|}{ BRL Subjects $(n=5)$} & \multicolumn{5}{|c|}{ PRL Subjects $(n=5)$} \\
\hline & SA & A & NC & D & SD & SA & A & NC & D & SD \\
\hline \multirow{5}{*}{$\begin{array}{l}\text { This liner adds noticeable weight to my } \\
\text { prosthesis. } \\
\text { This liner reduces pain in my residual limb. } \\
\text { This liner increases comfort during walking. } \\
\text { This liner increases "pistoning." } \\
\text { This liner increases the amount of energy } \\
\text { needed to walk. }\end{array}$} & 1 & 2 & 1 & 1 & - & - & 4 & - & 1 & - \\
\hline & 2 & 3 & - & - & - & 1 & 1 & 1 & 1 & 1 \\
\hline & 2 & 2 & - & 1 & - & 1 & 2 & - & 1 & 1 \\
\hline & - & 1 & 1 & 3 & - & - & - & - & 4 & 1 \\
\hline & 1 & 1 & 1 & 2 & - & 1 & - & - & 3 & 1 \\
\hline \multirow{3}{*}{$\begin{array}{l}\text { I am able to walk faster with this liner. } \\
\text { Walking feels smoother with this liner. } \\
\text { This liner decreases twisting between the } \\
\text { socket and my residual limb. }\end{array}$} & - & 3 & 2 & - & - & - & 2 & 2 & 1 & - \\
\hline & 1 & 2 & 1 & 1 & - & 1 & 2 & - & 2 & - \\
\hline & 1 & 1 & 1 & 2 & - & - & 2 & 2 & - & 1 \\
\hline \multirow{3}{*}{$\begin{array}{l}\text { I do not feel pressure in individual areas on } \\
\text { my residual limb. } \\
\text { This liner increases comfort during standing. } \\
\text { This liner decreases "pistoning." }\end{array}$} & 2 & 3 & - & - & - & - & 3 & 1 & - & 1 \\
\hline & 1 & 3 & - & 1 & - & 2 & 1 & - & 2 & - \\
\hline & - & 3 & 1 & 1 & - & 1 & 4 & - & - & - \\
\hline \multirow{4}{*}{$\begin{array}{l}\text { This liner makes my prosthesis more rigid. } \\
\text { This liner enables me to turn easier. } \\
\text { This liner compresses my limb uniformly. } \\
\text { This liner makes my residual limb too hot. }\end{array}$} & - & 2 & 2 & 1 & - & 1 & 2 & - & 2 & - \\
\hline & - & 3 & 2 & - & - & - & 2 & 2 & 1 & - \\
\hline & - & 1 & 2 & 2 & - & - & 2 & 1 & 2 & - \\
\hline & 1 & - & 2 & 2 & - & - & 1 & 1 & 3 & - \\
\hline \multirow{4}{*}{$\begin{array}{l}\text { This liner makes my knee harder to bend. } \\
\text { This liner increases perspiration of my } \\
\text { residual limb. } \\
\text { This liner reduces shock to my residual limb } \\
\text { during walking. } \\
\text { I like using this liner with my prosthesis. }\end{array}$} & 1 & 2 & 1 & 1 & - & 1 & 3 & - & 1 & - \\
\hline & 1 & - & 2 & 2 & - & - & 2 & 1 & 2 & - \\
\hline & 1 & 2 & 1 & 1 & - & 1 & 1 & - & 3 & - \\
\hline & 1 & 1 & - & 3 & - & 1 & 1 & 1 & 1 & 1 \\
\hline \multirow{2}{*}{ Which liner do you prefer? } & \multicolumn{2}{|c|}{$3 \mathrm{~mm}$} & \multirow{2}{*}{\multicolumn{3}{|c|}{$\begin{array}{c}9 \mathrm{~mm} \\
4\end{array}$}} & \multicolumn{2}{|c|}{$3 \mathrm{~mm}$} & \multirow{2}{*}{\multicolumn{3}{|c|}{$9 \mathrm{~mm}$}} \\
\hline & & & & & & & & & & \\
\hline
\end{tabular}

Figure 4.

Questionnaire was administered after subjects completed testing of both liners. Subjects were instructed to respond to series of statements using 5-point Likert scale to compare $9 \mathrm{~mm}$ liner to $3 \mathrm{~mm}$ liner condition. Numbers provided in table represent number of responses by each subgroup of subjects. Column headings represent following responses: strongly agree (SA), agree (A), no change/do not know (NC), disagree (D), and strongly disagree (SD). Shading denotes statements on which majority of subjects agreed or disagreed.

was used, pressure was only measured at locations on the posterior aspect of the residual limb, which tends to have greater bulk of soft tissue [19]. Thus, the compliant soft tissue in the measurement region may have produced peak pressures that were lower than those that would have occurred at sites with bony prominences, as are reported in this study. Nonetheless, the peak interface pressure values that were observed in this study are not unprecedented. Another study of PTB socket pressure found a stance-phase peak pressure of $417 \mathrm{kPa}$ at the patellar tendon [17]. The PTB socket is designed to load the patellar tendon, so this higher reported pressure in that location is not particularly surprising. The range and variability in pressure measurements reported in the literature could potentially be explained by differences in the prosthetic componentry, testing equipment, and positioning of the sensors. Only one of these studies was performed with a capacitive sensor array comparable to the one that was used in this current study [18]. The other studies used force-sensing resistors $[17,19]$ or diaphragm deflection strain-gauge sensors [20-21]. Additionally, the positioning of the pressure sensors was inconsistent between studies; in some protocols the sensors were placed between the residual limb and the prosthetic liner [19-20]; in others between the interface material (sock/ liner) and the socket [18,21]; and in one investigation without liners, they were placed directly between the residual limb and the socket [17].

There are some preliminary indications that the compliance provided by the thicker gel liner produced a more uniform pressure distribution across the subjects' residual limbs. Compared with the $3 \mathrm{~mm}$ liner, the $9 \mathrm{~mm}$ liner significantly reduced peak pressures at the $\mathrm{sFH}$ for both limb-type subgroups. Though not statistically significant, individual results from the BRL subjects (Table 2) demonstrated that the $9 \mathrm{~mm}$ liner consistently produced lower peak pressures over the sPT, and in 60 percent of the BRL subjects the peak pressures were lower over the sDT. Nonetheless, the small size of the BRL subsample and the relatively large SD in the pressure data do not allow a reliable statistical analysis to be conducted, so we are unable to draw definitive conclusions about the reduction of pressure at these sites on the residual limb. For the BRL group, the increased compliance provided by the $9 \mathrm{~mm}$ liner may have contributed to a redistribution of pressure over the residual limb. Presumably, establishing a more uniform pressure distribution over the residual limb would require that the reduction in peak pressures observed at high load-bearing sites be accompanied by an increase in peak pressures in those regions of the residual limb with a greater bulk of soft tissue. However, additional studies are required that will collect pressure measurement data over a greater portion of the residual limb to produce the necessary evidence to conclusively determine whether thicker gel liners create a more uniform pressure distribution. In the PRL subjects, a fairly uniform distribution may have already been provided by the bulk of soft tissue present at this interface, rendering the compliance provided by the thicker gel liner redundant.

The reduction in peak pressure over the sFH recorded with the $9 \mathrm{~mm}$ liner appeared to have had little effect on the gait characteristics of research subjects. The only gait parameter that changed significantly between liner conditions was an increased prosthetic-side vertical GRF peak during weight acceptance ( $p=0.02$, Wilcoxon signedranks test) observed with the $9 \mathrm{~mm}$ liner. This finding contradicts the stated hypothesis, which predicted a smaller prosthetic-side GRF peak would be observed with the thicker gel liner. This change in GRF loading peak 
cannot be explained by walking speed alone, since no statistically significant difference in speed existed between liners. A potential explanation for an increased GRF during walking is that the thicker liner may have impeded force and vibration transmission through the prosthesis to the subjects' residual limbs, reducing the sensory feedback that the subjects may rely on to determine when initial contact of the prosthetic foot occurs. Therefore, the subjects may have stepped more forcefully to increase sensory feedback.

The freely selected walking speed was not increased with the thicker liner as we had hypothesized. However, the subjects' perception of greater instability while walking with the thicker liner is one potential factor that may have affected walking speed. The subjects may have perceived greater instability with the thicker liner, which may reflect their unfamiliarity with having the increased compliance at the limb-socket interface. The interface between the residual limb and the prosthetic socket may be considered a pseudo-arthrosis, as it allows relative rotations and translations when experiencing loads (as in walking). The limb and the socket may move with respect to one another, and this rotational and translational motion may be magnified by increased compliance from the combined compression of anatomical soft tissue and gel liner during activity. The thicker gel liner could potentially allow greater relative motion between the residual limb and the socket, which subjects may have perceived as less stable or secure. This sensation may have been particularly noticeable for the BRL group, who did not have as much anatomical compliance (i.e., soft tissue bulk) on their residual limbs as the PRL subjects. Only one BRL subject was wearing a $9 \mathrm{~mm}$ liner when enrolled in this study. Therefore, increases in walking speed and improvements in other gait parameters associated with the reduced peak pressures observed with the $9 \mathrm{~mm}$ liner may have been offset by perceptions of instability within the socket. Longer accommodation times may have permitted the subjects to become more confident while walking with the thicker liner, leading to the changes in gait biomechanics that were originally anticipated.

Additionally, while walking speed may be influenced by shock transmission [26], it may also reflect the energy cost of walking [27]. The increased weight of the thicker gel liner may have increased the energy required to walk, thereby offsetting any potential benefits that could be gained from the lower peak pressure transmission to the residual limb. However, this explanation seems unlikely; while the $9 \mathrm{~mm}$ liner was approximately 2.15 times heavier than the $3 \mathrm{~mm}$ liner, the difference in mass was only about $0.341 \mathrm{~kg}$. Lehmann et al. investigated the effect of adding mass to transtibial prostheses and showed that when mass was added to the proximal end of the prosthesis (as was the case with the thicker liner), energy expenditure for level walking was not significantly affected [28]. The average difference in mass during Lehmann et al.'s study was approximately $1.5 \mathrm{~kg}$, compared with the $0.341 \mathrm{~kg}$ found in this study. Therefore, it seems unlikely that the weight of the thicker liner substantially increased subjects' energy expenditure. Nonetheless, other potential factors, such as muscular co-contraction by subjects during prosthetic stance phase due to their perception of instability, may also have influenced energy expenditure during this study. Additional studies of this type that measure energy expenditure and collect electromyography data are required to identify and better understand the various consequences of fitting transtibial prosthesis users with different thicknesses of gel liners.

Subjects generally expressed strong preferences between the two liners. The BRL group's overwhelming preference for the $9 \mathrm{~mm}$ liner is interesting, particularly since they did not experience much alteration in gait performance between the two liners. All BRL subjects experienced a statistically significant reduction in the peak pressure over their sFH with the thicker liner. Though not statistically significant, they also had reduced peak pressure over the sPT, and the majority of BRL subjects also had a reduction of pressures over the sDT. Though speculative, this finding suggests that a prosthesis user's perception of comfort may be heavily influenced by the intrasocket pressure distribution they experience while walking. On the other hand, the PRL subjects expressed a mixed preference between the two liners. However, the magnitudes of the peak pressures that were recorded for both liner conditions in the PRL group were generally much lower than those experienced in the BRL group, possibly indicating that the pressure distributions on their residual limbs were more uniform because of the increased bulk of soft tissue. Thus, some of the subjects in the PRL group may not have experienced a redistribution of pressure with the $9 \mathrm{~mm}$ liner, and other significant concerns, such as their perceptions of increased temperature and bulk of the thicker liner, were more influential on their preference decision.

A significant limitation of this study pertains to the variability in socket fit. Because socket fit can play a crucial role in pressure distribution at the limb-socket interface, the study protocol was designed to reduce 
inconsistency in this procedure by digitally scanning subjects' residual limbs while they were wearing the liner and fabricating the sockets with computer-aided design/manufacturing technology. Despite this effort to standardize socket fit, further modifications to the individual sockets were often necessary to ensure a comfortable fit. These modifications - mainly bony relief - were performed on an individual basis at the discretion of the study prosthetist. The $3 \mathrm{~mm}$ socket generally required more frequent modifications than the $9 \mathrm{~mm}$ socket, and bony relief was occasionally required in those areas where pressures were measured. As a result, the final socket shape may have been altered from that originally fabricated, potentially changing the distribution of pressure on the residual limb. However, no bony relief was provided in the sockets for the $9 \mathrm{~mm}$ liner without a corresponding modification in that subject's $3 \mathrm{~mm}$ socket, so the modifications that were performed for the $3 \mathrm{~mm}$ liner sockets may have resulted in an artificially low peak pressure reading. Thus, reductions in peak pressure observed with the $9 \mathrm{~mm}$ liner may be directly attributed to the additional compliance of the thicker gel liner. Nevertheless, it is recommended that in future studies of this type, a three-dimensional scan be acquired of the initial and final socket shapes to quantify the differences in volume and cross-sectional areas that occur as a result of socket modifications.

Another limitation of this study is that the order of testing for the two liner conditions was not prospectively randomized. The protocol was designed so that subjects would test the $3 \mathrm{~mm}$ liner first to establish this condition as baseline. However, difficulty with fitting some subjects with the $3 \mathrm{~mm}$ liner necessitated a change in order for four subjects. Because the order was not randomly assigned, the testing order may have an inherent bias that was unanticipated and not accounted for in the results, such as limbs having more prominent bony protuberances or greater sensitivity to pain or pressure. However, the subjects who tested the $9 \mathrm{~mm}$ liner first were not determined to be substantially different in age, weight, or limb-type group compared with those subjects who tested the $3 \mathrm{~mm}$ liner first or to have different current prosthetic prescriptions. Also, the pseudo-random testing order that transpired may have avoided any potential order effects that could have occurred if the $3 \mathrm{~mm}$ liner was always tested before the $9 \mathrm{~mm}$ liner.

The pressure measurement system that was used for this study may have inadvertently influenced the peak pressure results. The interface between the residual limb and the prosthetic socket is a harsh environment because of the high interface pressures and stresses, temperature, and curvature of the socket with respect to the residual limb. The capacitive sensors used in this experiment have a manufacturer-reported error of 5 percent and a resolution of 2-7 kPa. However, a previous study of a prototypical version of these sensors reported their error as being $9.96 \pm 9.1$ percent when they were evaluated on a simulated residual limb [29]. In addition to measurement accuracy, the sensor thickness may also have affected the results. During limb digitization and socket fitting, no accommodation was made for the thickness of the pressure sensors. Therefore, the socket fit may have been altered by the incorporation of the pressure sensors. The sensors used within this study were $1 \mathrm{~mm}$ thick, compared with the liner thicknesses of $3 \mathrm{~mm}$ and $9 \mathrm{~mm}$. The relative thickness of the $9 \mathrm{~mm}$ liner may have prevented the development of localized high pressures over the sensors. However, the $1 \mathrm{~mm}$ thickness of the sensors is more significant in the case of the $3 \mathrm{~mm}$ liner, so they may have had a more substantial influence on socket fit, patient comfort, and pressure measurements. This effect could be even more dramatic over stiff, bony prominences such as the fibular head, and may be partially responsible for the elevated pressure values observed in this study compared with those reported in the literature. However, the socket fit was standardized between subjects as much as possible and the same sensors were used for both liner conditions. Even if slightly elevated peak pressures occurred with the $3 \mathrm{~mm}$ liner because of sensor thickness, the absence of this result for the $9 \mathrm{~mm}$ liner condition could indicate that the thicker liner was able to redistribute elevated peak pressures to adjacent areas of the residual limb.

The number of sites on the residual limb at which pressure was measured was limited in this study. Pressure data were acquired at only three specific locations on the subjects' residual limbs because of problems that developed with two other sensor arrays. To provide more compelling evidence that a thicker liner is able to distribute pressure more uniformly over the residual limb compared with a thinner liner, researchers should implement experimental protocols that incorporate pressure sensors over more sites on the residual limb, including regions with soft tissues and bony landmarks. Additionally, the accommodation period given for the experimental prosthesis may not have been sufficient to effect significant changes in gait parameters, particularly in the BRL subjects who 
were not as accustomed to the increased compliance at the limb-socket interface. Subjects were asked to wear the experimental prosthesis as much as possible during the 2week accommodation period, but the extent of each subjects' fluency with the prosthesis was not quantified. While a 2-week accommodation period is fairly common in studies of prosthetic components for unilateral transtibial amputees, a minimum recommended length of time for accommodation with these gel liners is unknown. Had a longer accommodation period been part of this protocol, the subjects may have become better accustomed to the increased compliance at the residual limb-prosthetic socket interface and their gait biomechanics may have changed to better reflect the benefit they derived from the shock-absorbing characteristics of the liner.

Finally, shear stresses were not recorded in this study. Shear stresses are an important consideration in lowerlimb amputees because they can produce tissue breakdown and can contribute to other skin problems that occur at the residual limb. Many studies have demonstrated that skin irritation, sores, blisters, and chafing are frequent occurrences in persons with lower-limb amputation that can adversely affect quality of life [30-33]. For this reason, shear stresses on the residual limb and various factors that may affect them have been evaluated in the literature [21-22]. Prosthetic gel liners may influence shear stresses on the residual limb by providing sufficient compliance to attenuate peak stresses parallel to the surface of the limb through deformation of the liner material. Additionally, computational modeling has indicated that use of a gel liner can influence shear stresses at the residual limb-prosthetic socket interface [34]. Future work correlating gel liner thickness with shear stress distribution on the residual limb may provide additional insight on the mechanisms that contribute to the comfort perceived by prosthesis users at the limb-socket interface.

Thicker gel liners (6 $\mathrm{mm}$ or greater) are often prescribed to patients for reasons of cushioning and comfort; however, these prescriptions have been largely based on the prosthetist's anecdotal observation, experience and intuition, patient feedback, and manufacturer recommendations. This study has attempted to evaluate the effect that gel liners of different thickness have on pressures at the residual limb-liner interface and on the gait characteristics of persons with unilateral transtibial amputations. The findings of this study-in terms of an overall reduction in sFH pressure-support subjective claims of increased comfort with thicker gel liners. However, a small but unexpected increase in the loading peak of the vertical GRF could indicate that the thicker liner reduces transmission of vibrotactile information from the prosthetic socket to the residual limb that users may subconsciously rely on during walking. Additionally, gel liners have been associated with increased perspiration and heat at the residual limb-socket interface [16]. Three subjects in the current study commented on temperature issues arising from the $9 \mathrm{~mm}$ liner. The perceived temperature at the residual limb-socket interface has been implicated as a major determinant of comfort, and liner material type has been identified as a possible factor that influences residual limb skin temperature [35]. Future work aimed at clearly establishing which liner thickness is most appropriate for particular residual limb types should include protocols that are designed to enable a thorough and comprehensive evaluation of all aspects of the residual limb condition within the socket, including quantitative measures of temperature and perspiration at the limb-liner interface, in addition to pressure recordings acquired at multiple sites over the limb to permit pressure distributions to be determined. Additionally, an evaluation of peak pressures during activities other than straight, level walking (e.g., turning, running, inclined surfaces, and stairs) could provide critical information on how liner thickness may influence the performance of activities of daily living.

\section{CONCLUSIONS}

Gel liner thickness was found to affect certain variables related to gait, pressure distribution at the residual limb-prosthetic liner interface, and subject perception of comfort. The thicker liner significantly reduced peak pressure over the fibular head in all subjects. A statistically significant increase in the magnitude of the vertical GRF loading peak was also observed with the $9 \mathrm{~mm}$ liner, potentially due to reduced somatosensory feedback to the residual limb during loading response phase. Nevertheless, the thicker liner increased perceived comfort and was preferred over the thinner liner by a majority of the study participants. A more comprehensive evaluation of the pressure distribution within the residual limb-prosthetic socket interface that takes into consideration other factors relating to subject perceptions of comfort, such as pistoning, perspiration, and temperature, may shed further light on appropriate liner thickness prescriptions. 


\section{ACKNOWLEDGMENTS}

\section{Author Contributions:}

Study concept and design: S. Gard, R. Stine, A. Hansen.

Acquisition of data: E. Boutwell, R. Stine, A. Hansen, K. Tucker. Analysis and interpretation of data: E. Boutwell, R. Stine, A. Hansen, S. Gard.

Drafting of manuscript: E. Boutwell.

Critical revision of manuscript for important intellectual content: E. Boutwell, R. Stine, A. Hansen, K. Tucker, S. Gard.

Study supervision: S. Gard.

Financial Disclosures: The authors have declared that no competing interests exist.

Funding/Support: This material was based on work supported by the VA, Office of Research and Development, Rehabilitation Research and Development Service (grant A3518R) and administered by the JBVAMC, Chicago, Illinois.

Additional Contributions: The authors would like to thank Mark Edwards, CP, and Margrit Meier, PhD, CPO, for their contributions to this project. The authors also acknowledge the use of the JBVAMC MARL, Chicago, Illinois.

Institutional Review: Each subject signed a consent form approved by the Northwestern University Institutional Review Board prior to participation in this study.

Participant Follow-Up: The authors do not plan to inform participants of the publication of this study. However, participants have been encouraged to check Capabilities (NUPOC's quarterly newsletter) and Web site for updated publications.

Disclaimer: The views expressed in this article are those of the authors and do not necessarily reflect the position or policy of the VA or the U.S. Government.

\section{REFERENCES}

1. Marks LJ, Michael JW. Science, medicine, and the future: Artificial limbs. BMJ. 2001;323(7315):732-35.

PMID:11576982

http://dx.doi.org/10.1136/bmj.323.7315.732

2. Voloshin A, Wosk J. An in vivo study of low back pain and shock absorption in the human locomotor system. J Biomech. 1982;15(1):21-27. PMID:6460773 http://dx.doi.org/10.1016/0021-9290(82)90031-8

3. Radin EL, Parker HG, Pugh JW, Steinberg RS, Paul IL, Rose RM. Response of joints to impact loading. 3. Relationship between trabecular microfractures and cartilage degeneration. J Biomech. 1973;6(1):51-57.

PMID:4693868

http://dx.doi.org/10.1016/0021-9290(73)90037-7

4. Klute GK, Kallfelz CF, Czerniecki JM. Mechanical properties of prosthetic limbs: Adapting to the patient. J Rehabil Res Dev. 2001;38(3):299-307. PMID:11440261

5. Kaufman KR, Sutherland DH. Kinematics of normal human walking. In: Rose J, Gamble JG, editors. Human walking. 3rd ed. Philadelphia (PA): Lippincott Williams \& Wilkins; 2006. p. 33-51.

6. Gard SA, Childress DS. The influence of stance-phase knee flexion on the vertical displacement of the trunk during normal walking. Arch Phys Med Rehabil. 1999;80(1): 26-32. PMID:9915368 http://dx.doi.org/10.1016/S0003-9993(99)90303-9

7. Breakey J. Gait of unilateral below-knee amputees. Orthot Prosthet. 1976;30(3):17-24.

8. Michaud SB, Gard SA, Childress DS. A preliminary investigation of pelvic obliquity patterns during gait in persons with transtibial and transfemoral amputation. J Rehabil Res Dev. 2000;37(1):1-10. PMID:10847567

9. Casillas JM, Dulieu V, Cohen M, Marcer I, Didier JP. Bioenergetic comparison of a new energy-storing foot and SACH foot in traumatic below-knee vascular amputations. Arch Phys Med Rehabil. 1995;76(1):39-44.

PMID:7811172

http://dx.doi.org/10.1016/S0003-9993(95)80040-9

10. Lehmann JF, Price R, Boswell-Bessette S, Dralle A, Questad K. Comprehensive analysis of dynamic elastic response feet: Seattle Ankle/Lite Foot versus SACH foot. Arch Phys Med Rehabil. 1993;74(8):853-61. PMID:8347071 http://dx.doi.org/10.1016/0003-9993(93)90013-Z

11. Torburn L, Perry J, Ayyappa E, Shanfield SL. Below-knee amputee gait with dynamic elastic response prosthetic feet: A pilot study. J Rehabil Res Dev. 1990;27(4):369-84. PMID:2089148 http://dx.doi.org/10.1682/JRRD.1990.10.0369

12. Barr AE, Siegel KL, Danoff JV, McGarvey CL 3rd, Tomasko A, Sable I, Stanhope SJ. Biomechanical comparison of the energy-storing capabilities of SACH and Carbon Copy II prosthetic feet during the stance phase of gait in a person with below-knee amputation. Phys Ther. 1992;72(5): 344-54. PMID:1631203

13. Gard SA, Konz RJ. The effect of a shock-absorbing pylon on the gait of persons with unilateral transtibial amputation. J Rehabil Res Dev. 2003;40(2):109-24.

PMID:15077637 http://dx.doi.org/10.1682/JRRD.2003.03.0109

14. Miller LA, Childress DS. Analysis of a vertical compliance prosthetic foot. J Rehabil Res Dev. 1997;34(1):52-57. PMID:9021625

15. Fergason J, Smith DG. Socket considerations for the patient with a transtibial amputation. Clin Orthop Relat Res. 1999; (361):76-84. PMID:10212599 http://dx.doi.org/10.1097/00003086-199904000-00011

16. Hatfield AG, Morrison JD. Polyurethane gel liner usage in the Oxford Prosthetic Service. Prosthet Orthot Int. 2001; 25(1):41-46. PMID:11411004 http://dx.doi.org/10.1080/03093640108726567

17. Convery P, Buis AW. Socket/stump interface dynamic pressure distributions recorded during the prosthetic stance 
phase of gait of a trans-tibial amputee wearing a hydrocast socket. Prosthet Orthot Int. 1999;23(2):107-12. PMID:10493137

18. Dou P, Jia X, Suo S, Wang R, Zhang M. Pressure distribution at the stump/socket interface in transtibial amputees during walking on stairs, slope and non-flat road. Clin Biomech (Bristol, Avon). 2006;21(10):1067-73.

PMID:16919376

http://dx.doi.org/10.1016/j.clinbiomech.2006.06.004

19. Beil TL, Street GM, Covey SJ. Interface pressures during ambulation using suction and vacuum-assisted prosthetic sockets. J Rehabil Res Dev. 2002;39(6):693-700. PMID:17943671

20. Sonck WA, Cockrell JL, Koepke GH. Effect of liner materials on interface pressures in below-knee prostheses. Arch Phys Med Rehabil. 1970;51(11):666-69. PMID:5501936

21. Sanders JE, Zachariah SG, Baker AB, Greve JM, Clinton C. Effects of changes in cadence, prosthetic componentry, and time on interface pressures and shear stresses of three trans-tibial amputees. Clin Biomech (Bristol, Avon). 2000; 15(9):684-94. PMID:10946102 http://dx.doi.org/10.1016/S0268-0033(00)00026-7

22. Sanders JE, Zachariah SG, Jacobsen AK, Fergason JR. Changes in interface pressures and shear stresses over time on trans-tibial amputee subjects ambulating with prosthetic limbs: Comparison of diurnal and six-month differences. J Biomech. 2005;38(8):1566-73. PMID:15958212 http://dx.doi.org/10.1016/j.jbiomech.2004.08.008

23. Silver-Thorn MB, Steege JW, Childress DS. A review of prosthetic interface stress investigations. J Rehabil Res Dev. 1996;33(3):253-66. PMID:8823673

24. Rolock JS, Tucker K, editors. Squirt shape-Development of an automated fabrication technique to enhance prosthetics CAD/CAM. 2nd National VA Rehabilitation Research \& Development Conference; 2000 Feb 20-22; Arlington, VA.

25. Kadaba MP, Ramakrishnan HK, Wootten ME. Measurement of lower extremity kinematics during level walking. J Orthop Res. 1990;8(3):383-92. PMID:2324857 http://dx.doi.org/10.1002/jor.1100080310

26. Ratcliffe RJ, Holt KG. Low frequency shock absorption in human walking. Gait Posture. 1997;5(2):93-100. http://dx.doi.org/10.1016/S0966-6362(96)01077-6.

27. Waters RL, Perry J, Antonelli D, Hislop H. Energy cost of walking of amputees: The influence of level of amputation. J Bone Joint Surg Am. 1976;58(1):42-46. PMID:1249111

28. Lehmann JF, Price R, Okumura R, Questad K, de Lateur BJ, Négretot A. Mass and mass distribution of below-knee prostheses: Effect on gait efficacy and self-selected walking speed. Arch Phys Med Rehabil. 1998;79(2):162-68. PMID:9473997 http://dx.doi.org/10.1016/S0003-9993(98)90293-3
29. Polliack AA, Craig DD, Sieh RC, Landsberger S, McNeal DR. Laboratory and clinical tests of a prototype pressure sensor for clinical assessment of prosthetic socket fit. Prosthet Orthot Int. 2002;26(1):23-34. PMID:12043923 http://dx.doi.org/10.1080/03093640208726618

30. Dillingham TR, Pezzin LE, MacKenzie EJ, Burgess AR. Use and satisfaction with prosthetic devices among persons with trauma-related amputations: A long-term outcome study. Am J Phys Med Rehabil. 2001;80(8):563-71. PMID:11475475 http://dx.doi.org/10.1097/00002060-200108000-00003

31. Hagberg K, Brånemark R. Consequences of non-vascular trans-femoral amputation: A survey of quality of life, prosthetic use and problems. Prosthet Orthot Int. 2001;25(3): 186-94. PMID:11860092 http://dx.doi.org/10.1080/03093640108726601

32. Legro MW, Reiber G, del Aguila M, Ajax MJ, Boone DA, Larsen JA, Smith DG, Sangeorzan B. Issues of importance reported by persons with lower limb amputations and prostheses. J Rehabil Res Dev. 1999;36(3):155-63. PMID:10659798

33. Hoaglund FT, Jergesen HE, Wilson L, Lamoreux LW, Roberts R. Evaluation of problems and needs of veteran lowerlimb amputees in the San Francisco Bay Area during the period 1977-1980. J Rehabil Res Dev. 1983;20(1):57-71. PMID:6887067

34. Silver-Thorn MB, Childress DS. Generic, geometric finite element analysis of the transtibial residual limb and prosthetic socket. J Rehabil Res Dev. 1997;34(2):171-86. PMID:9108344

35. Peery JT, Ledoux WR, Klute GK. Residual-limb skin temperature in transtibial sockets. J Rehabil Res Dev. 2005; 42(2):147-54. PMID:15944879 http://dx.doi.org/10.1682/JRRD.2004.01.0013

Submitted for publication on June 24, 2010. Accepted in revised form May 16, 2011.

This article and any supplementary material should be cited as follows:

Boutwell E, Stine R, Hansen A, Tucker K, Gard S. Effect of prosthetic gel liner thickness on gait biomechanics and pressure distribution within the transtibial socket. J Rehabil Res Dev. 2012;49(2):227-40.

http://dx.doi.org/10.1682/JRRD.2010.06.0121

ResearcherID: Steven Gard, PhD: D-9935-2011

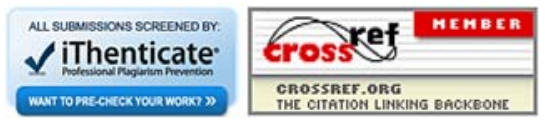

